\title{
Effect of Th1/Th2 cytokine administration on proinflammatory SKOV-3 cell activation
}

\author{
Aleksandra Mielczarek-Palacz ${ }^{1}$, Justyna Sikora ${ }^{1}$, Zdzisława Kondera-Anasz ${ }^{1}$, Patrycja Mickiewicz ${ }^{2}$, \\ Adam Mickiewicz ${ }^{1}$
}

\begin{abstract}
${ }^{1}$ Department of Immunology and Serology, School of Pharmacy with the Division of Medical Analytics in Sosnowiec, Medical University of Silesia, Katowice, Poland ${ }^{2} \mathrm{X}$-ray Microtomography Laboratory, Department of Biomedical Computer Systems, Institute of Computer Science, Faculty of Computer Science and Material Science, University of Silesia, Katowice, Poland
\end{abstract}

Submitted: 10 October 2014

Accepted: 3 January 2015

Arch Med Sci 2016; 12, 6: 1337-1347

DOI: $10.5114 /$ aoms.2015.53143

Copyright @ 2015 Termedia \& Banach

\section{Abstract}

Introduction: Interleukin(IL)-1 $\beta$, IL-6 and IL-12 might associate with inflammatory processes in a tumor progression and create a specific microenvironment for tumor growth. The aim of the study was to assess whether the Th1 and Th2 type cytokines, such as IL-2 and IL-10, affect ovarian carcinoma continuous cell line (SKOV-3) pro-inflammatory activation.

Material and methods: SKOV-3 ovarian cells and peripheral blood mononuclear cells (PBMCs) were stimulated by IL-2 and IL-10. Additionally, SKOV-3 ovarian cells and PBMCs were co-cultured together. Proinflammatory activation of cancer cells was evaluated by measurement of IL-1 $\beta$ and IL- 6 levels in culture fluid after $72 \mathrm{~h}$ of incubation.

Results: SKOV-3 cells and PBMCs secreted IL-1 $\beta$ and IL-6. After stimulation by IL-2 and IL-10, secretion of studied parameters was changed in a dose-dependent manner. The addition of a higher IL-2 level gave rise to an increase of IL-1 $\beta$, IL- 6 and IL-12 secretion in SKOV-3 cells. Stimulation by IL-10 increased only IL- $1 \beta$ secretion in SKOV-3 cells. However, IL-6 secretion decreased after stimulation with $25 \mathrm{ng} / \mathrm{ml} \mathrm{IL-10}$. Activatory effects of IL-2 and inhibitory effects of IL-10 in co-culture of SKOV-3 and PBMCs were observed. Conclusions: Our results suggested that Th1/Th2 type of cytokines might influence pro-inflammatory activation of SKOV-3 ovarian cells. Co-cultures of SKOV-3 and PBMCs showed significant changes in cross-talk between cancer and immune cells.

Key words: IL-1 $\beta$, IL-6, IL-12, ovarian cancer SKOV-3 cells, Th1-Th2 cytokines.

\section{Introduction}

Ovarian cancer is a highly lethal gynecological cancer for which overall prognosis has remained poor over the past few decades [1]. It is the fourth leading cause of all deaths due to malignant neoplasms in women, after cancer of the breast, lung and bowel [2]. Two-thirds of patients present with advanced-stage disease, stage III and IV bf FIGO, and the majority will suffer recurrence of disease, require ongoing treatment, and eventually succumb to chemotherapy-resistant disease [3].

The pathophysiology of ovarian cancer is complex and still poorly understood. The growth and proliferation of tumor require the presence
Corresponding author:

Aleksandra Mielczarek-Palacz Department of Immunology and Serology Medical University of Silesia 8 Jedności St 41-200 Sosnowiec, Poland Phone/fax: +48 323641240 E-mail: apalacz@poczta. onet.pl 


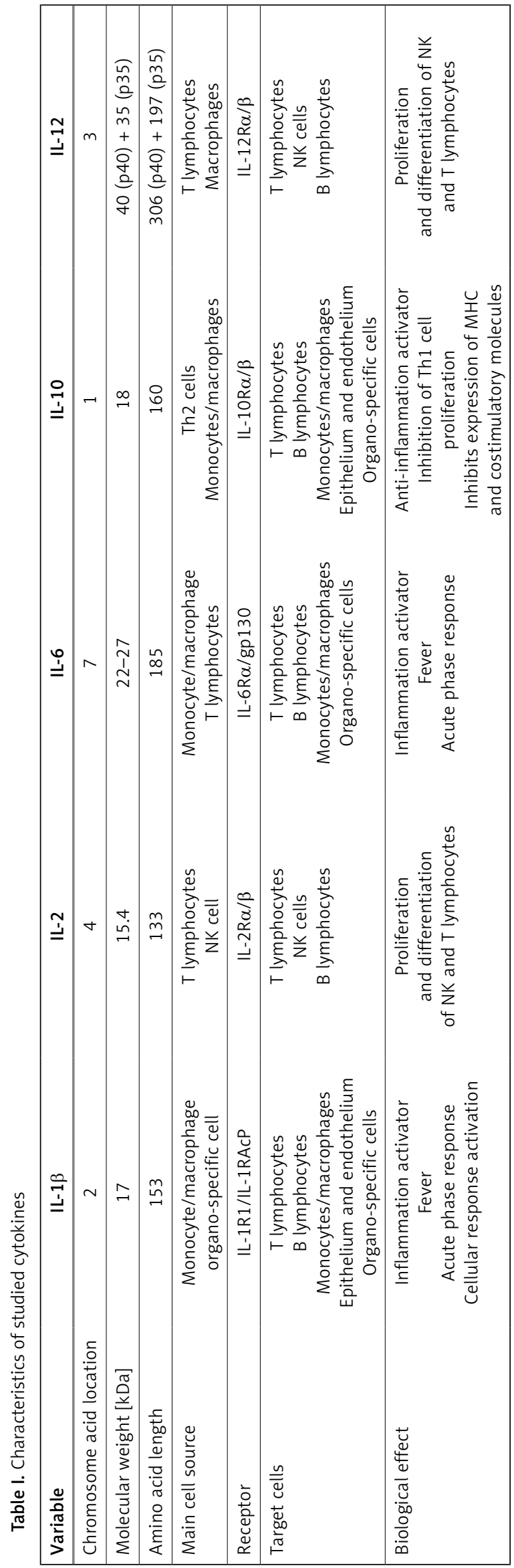

of new blood vessels and nutrients, and, on the other hand, a kind of tolerance on the part of the immune system [4-6]. In cancer patients there is a disorder in the functioning of the immune response, involved in the innate and adaptive immunity, including cytotoxic processes and antibody secretion, which may enhance susceptibility to growth and invasion of the cancer cells and consequently development of cancer $[7,8]$. One mechanism which is involved in ovarian cancer maintenance is the imbalance in the $\mathrm{T}$ helper 1 (Th1) and the T helper 2 (Th2) response, resulting in impairment of processes controlling homeostasis in the tumor microenvironment, such as inflammation and angiogenesis [9]. Diverting the immune response from Th1, including interleukin (IL)-2 and IL-12, towards the Th2 (IL-4, IL-10) response is considered to be one of the escape mechanisms of ovarian cancer. The Th1 type immune response stimulates cellular immunity by activating macrophages and dendritic cells and recruiting CD8+ cytotoxic T-cells (Tc) and natural killer (NK) cells. The Th2 immune response inhibits cell-mediated immunity and favors the humoral immune response. The generation of either a Th1-type or Th2-type response depends on the balance between cytokines [10]. There is growing evidence that $T$ cell activation and its cytokine expression play a key role in impairment and deregulation of the immune anti-tumor and inflammation response [11].

The tumor environment in which ovarian carcinoma develops has been described as one enriched with a broad spectrum of proinflammatory cytokines and chemokines. In particular, several of these cytokines, such as IL-1 $\beta$, IL- 6 and IL-12, are produced by the tumor itself, besides stimulating cancer cell growth [12]. Cytokines produced by these cells are responsible not only for the induction of biological effects, but also for the creation of a specific microenvironment that also can promote cell proliferation and tumor growth and affects the severity of inflammation. As is well known, Th1/Th2 cytokine unbalanced production might predispose to changes in proinflammatory cytokine secretion [13]. Characteristics of the studied cytokines are shown in Table I.

Therefore, the aim of the study was to assess the mechanisms by which Th1 and Th2 cytokines, such as IL-2 and IL-10, affect ovarian carcinoma continuous cell line (SKOV-3) pro-inflammatory activation. For this purpose the levels of IL-1 $\beta, I L-6$ and IL-12, as major proinflammatory cytokines, in supernatant of culture were measured. In addition, the impact of the microenvironment of peripheral blood mononuclear cells (PBMCS) on SKOV-3 activity was examined in this study. 


\section{Material and methods}

\section{Media and reagents}

McCoy's 5A Modified Medium with L-Glutamine and Heat-Inactivated Fetal Bovine Serum (FBS) were purchased from American Type Culture Collection (Rockville, MD, USA). Dulbecco's Modified Eagle Medium (DMEM) with $4.5 \mathrm{~g} / \mathrm{l}$ glucose and L-glutamine was from BioWhittaker (Verviers, Belgium). Antibiotic/Antimycotic Solution containing $10000 \mathrm{U} / \mathrm{ml}$ penicillin, $10 \mathrm{mg} / \mathrm{ml}$ streptomycin, $25 \mathrm{\mu g} / \mathrm{ml}$ amphotericin and Lymphocyte Separation Medium (LSM) at a density of $1.077 \mathrm{~g} / \mathrm{ml}$ were from PAA Laboratories $\mathrm{GmbH}$ (Pasching, Austria). Phosphate-Buffered Saline (PBS) was from Regional Blood Bank in Katowice (Katowice, Poland). 0.4\% Trypan Blue Solution was from Sigma-Aldrich Chemical Corporation (Saint Louis, USA). Human IL-2 and IL-10 were from PeproTech Inc. (Rocky Hill, USA). Human IL-1 $\beta$ ELISA and Human IL-6 ELISA kits were from eBioscience (Vienna, Austria).

\section{SKOV-3 cell line}

The human ovarian cancer cell line SKOV-3 used in this study was purchased from ATCC (Rockville, MD, USA). The human ovarian cancer cell line SKOV-3 was cultured in McCoy's 5A Modified Medium with L-Glutamine (ATCC, Rockville, MD, USA) supplemented with $10 \%$ fetal bovine serum, $100 \mathrm{U} / \mathrm{ml}$ penicillin and $100 \mu \mathrm{g} / \mathrm{ml}$ streptomycin at $37^{\circ} \mathrm{C}$ in an atmosphere of $5 \% \mathrm{CO}_{2}$ in air. SKOV-3 cells were seeded at a density of $10^{6}$ cells/ml in 24-well plates (PAA Laboratories $\mathrm{GmbH}$, Pasching, Austria). Passaged 4-6 times, 70-80\% confluence SKOV-3 cells were used for further experiments. Cell viability was consistently $>95 \%$ as determined by trypan blue staining.

\section{PBMC culture}

PBMCs from healthy 34-year-old blood donor buffy coat (RCKiK, Katowice, Poland) were isolated by diluting with PBS, and next centrifuged at $400 \times \mathrm{g}$ for 20 min over LSM (PAA Laboratories $\mathrm{GmbH}$, Pasching, Austria). The interface cells were collected, pooled, and washed with PBS until the contaminating platelets were removed. PBMCs were suspended at a final concentration of $1 \times 10^{6}$ cells $/ \mathrm{ml}$ in DMEM supplemented with $10 \%$ heat-inactivated FBS serum (Mediatech Inc, Manassas VA), $100 \mathrm{U} / \mathrm{ml}$ penicillin, $100 \mu \mathrm{g} / \mathrm{ml}$ streptomycin, and at $37^{\circ} \mathrm{C}$ and $5 \% \mathrm{CO}_{2}$. Cell viability was consistently $>95 \%$ as determined by trypan blue staining.

\section{Stimulation with IL-2 or IL-10}

SKOV-3 cells and PBMCs were cultured in media alone as a basal culture or stimulated culture in media containing $\mathrm{IL}-2$ or $\mathrm{IL}-10$, reaching final concentrations of $10 \mathrm{ng} / \mathrm{ml}$ and $25 \mathrm{ng} / \mathrm{ml}$. Cell viability was tested before and after incubation in all experiments.

\section{Variations of co-cultures}

After $72 \mathrm{~h}$ of stimulation with IL-2 or IL-10, all cells were harvested and suspended in McCoy's medium with $10 \%$ FBS and $1 \%$ antibiotics at a density of $10^{6}$ cells $/ \mathrm{ml}$. Co-cultures were set up by mixing equal amounts of both types of cell suspensions. Three variants of co-cultures were conducted: (i) co-cultures of SKOV-3 cells stimulated with IL-2 or IL-10 and unstimulated PBMCs, (ii) co-cultures of unstimulated SKOV-3 cells and PBMCs stimulated with IL-2 or IL-10, (iii) co-cultures in which both types of cells were stimulated with IL-2 or IL-10. After 72 h, co-culture supernatants were collected and stored at $-80^{\circ} \mathrm{C}$ for further analysis.

\section{IL-1 $\beta$ and IL-6 measurements}

Levels of IL-1 $\beta$, IL- 6 and IL-12 in culture media were measured by standard cytokine-specific enzyme-linked immunosorbent assay (ELISA) using commercial kits from eBioscience (Vienna, Austria). All determinations were performed in duplicate, according to the manufacturer's instructions, for each sample at the same time, to prevent any possible changes due to freezing and thawing. The sensitivity of the kits was approximately $0.3 \mathrm{pg} / \mathrm{ml}$ for IL-1 $\beta, 0.92 \mathrm{pg} / \mathrm{ml}$ for IL-6 and $2.1 \mathrm{pg} / \mathrm{ml}$ for IL-12. The precision (intra-assay and interassay) did not exceed $8 \%$ for all the assays.

\section{Statistical analysis}

Results were expressed as a mean \pm SD. The differences between and among culture variants were evaluated using one-way ANOVAS, with Fisher's test as a post-hoc analysis. The differences between mean values were considered statistically significant at $p<0.05$.

\section{Results}

\section{SKOV-3 cell culture}

Results for SKOV-3 cell culture are shown in Figure 1 . All cytokines, IL-1 $\beta$, IL- 6 and also IL-12 were secreted by SKOV-3 cells. IL-1 $\beta$ secretion was higher after stimulation with IL-2 and IL-10 in comparison to the basal culture $(p<0.0001)$. Increasing the dose of IL-2 did not significantly affect the proinflammatory cytokine production. However, after stimulation with a higher IL-10 level, SKOV-3 cells produced significantly more IL-1 $\beta$ than in culture stimulated with $10 \mathrm{ng} / \mathrm{ml} \mathrm{IL-10}(p<0.0001)$, where- 


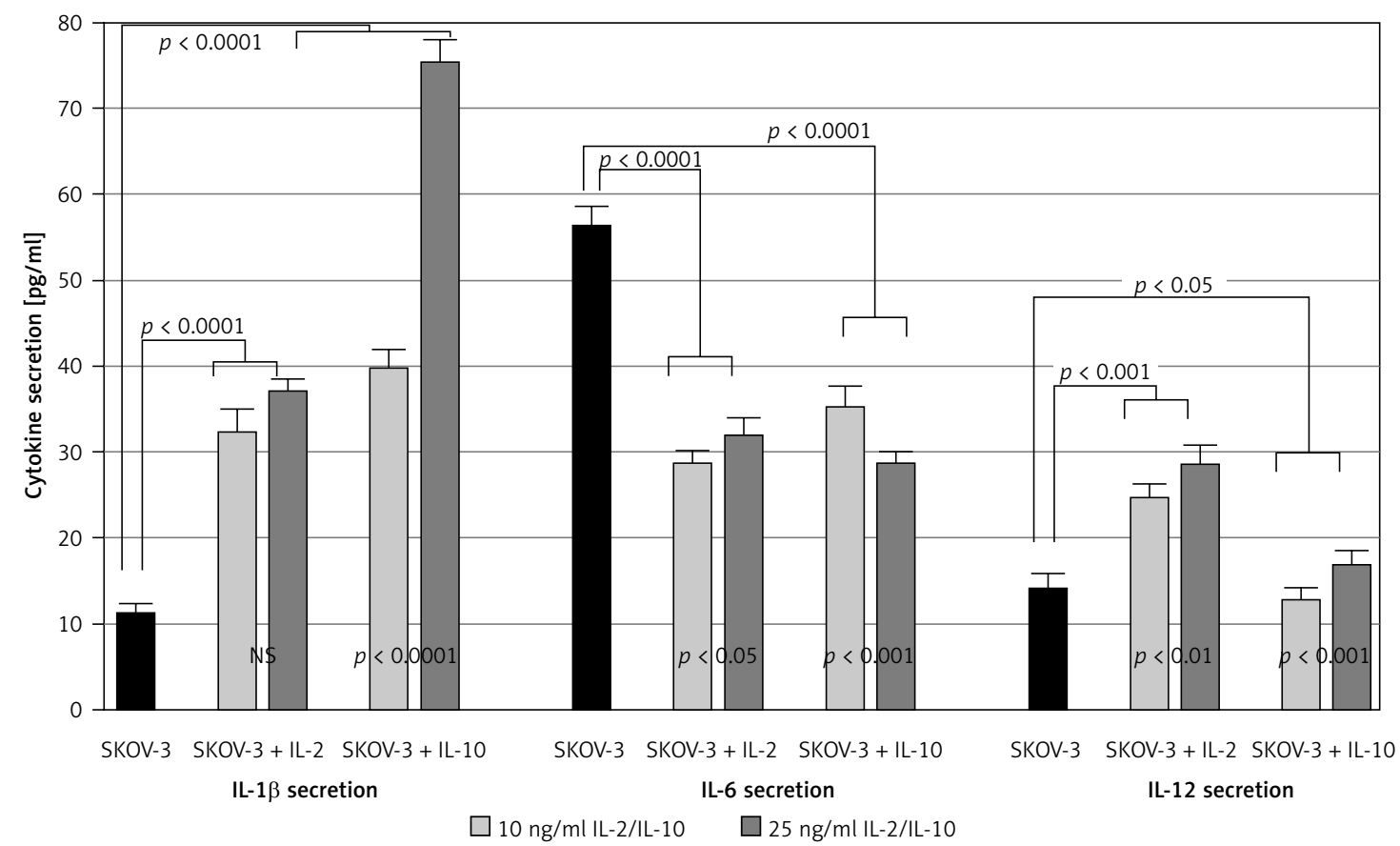

Figure 1. IL-1 $\beta$, IL-6 and IL-12 secretion by IL-2 or IL-10 activated SKOV-3 cells

as IL-6 secretion in basal culture was significantly higher compared to culture stimulated with either IL-2 or IL-10 ( $p<0.0001)$. There was no significant effect on IL- 6 production in relation to the concentration of both stimulatory factors in a dose-dependent manner. After stimulation by IL-2, secretion of IL-12 in a dose-dependent manner was higher than in the basal culture $(p<0.0001)$. However, IL-10 did not significantly change secretion of IL-12.

\section{PBMC culture}

All studied cytokines were secreted by PBMCs, but they produced more IL- 6 than IL-1 $\beta$ and the least IL-12. After stimulation with $10 \mathrm{ng} / \mathrm{ml} \mathrm{IL-2,}$ IL-1 $\beta$ secretion was significantly higher than in the basal culture and higher than after stimulation with $25 \mathrm{ng} / \mathrm{ml}(p<0.001)$. In addition, IL-1 $\beta$ level in culture medium after IL-10 stimulation was higher, but it was not dependent on IL-10 dose concentrations. IL- 6 secretion was lower after stimulation with $10 \mathrm{ng} / \mathrm{ml} \mathrm{IL}-2$ compared to the basal culture. After increasing the dose of IL-2, IL-6 production was significantly higher. The highest production of IL- 6 was observed after stimulation with IL-10. However, a different dose of IL-10 did not affect the IL- 6 production. When added to the culture, IL-2 secretion of IL-12 increased, but IL-10 slightly decreased production of IL-12. Results for the PBMC culture are shown in Figure 2.

\section{IL-1 $\beta$ secretion by co-culture}

IL-1 $\beta$ was secreted by co-culture of SKOV-3 and PBMC. SKOV-3 cells pre-stimulated with
IL-2 secreted less IL-1 $\beta$ in comparison with the basal co-culture. However, only stimulation with $10 \mathrm{ng} / \mathrm{ml}$ IL-10 significantly enhanced secretion of IL-1 $\beta$ compared to the basal co-culture ( $p<0.001)$. Co-culture of SKOV-3 cells and pre-stimulated PBMCs showed that only a higher dose of IL-10 led to significantly higher IL-1 $\beta$ secretion in comparison with unstimulated co-culture $(p<0.0001)$. Results for IL-1 $\beta$ secretion by co-cultures of SKOV-3 cells and PBMCs are shown in Figure 3.

\section{IL-6 secretion by co-culture}

Co-culture of SKOV-3 cells and PBMCs secreted IL-6. SKOV-3 cell and PBMC co-cultures pre-stimulated with IL-2 or IL-10 secreted less IL- 6 in comparison with the basal co-culture. However, only co-culture pre-stimulation of SKOV-3 cells with IL-2 and PBMCs significantly enhanced secretion of IL-6 in a dose-dependent manner. Results for IL-6 secretion by co-cultures of SKOV-3 cells and PBMCs are shown in Figure 4.

\section{IL-12 secretion by co-culture}

Co-culture of SKOV-3 cells and PBMCs secreted IL-12. Both SKOV-3 cells pre-stimulated with IL-2 and PBMCs in co-cultures secreted significantly more IL-12 in comparison with the basal co-culture $(p<0.0001)$. However, pre-stimulation of cells with IL-10 significantly inhibited production of IL- 6 in a dose-dependent manner $(p<0.0001)$. Results for IL- 6 secretion by co-cultures of SKOV-3 cells and PBMCs are shown in Figure 5. 


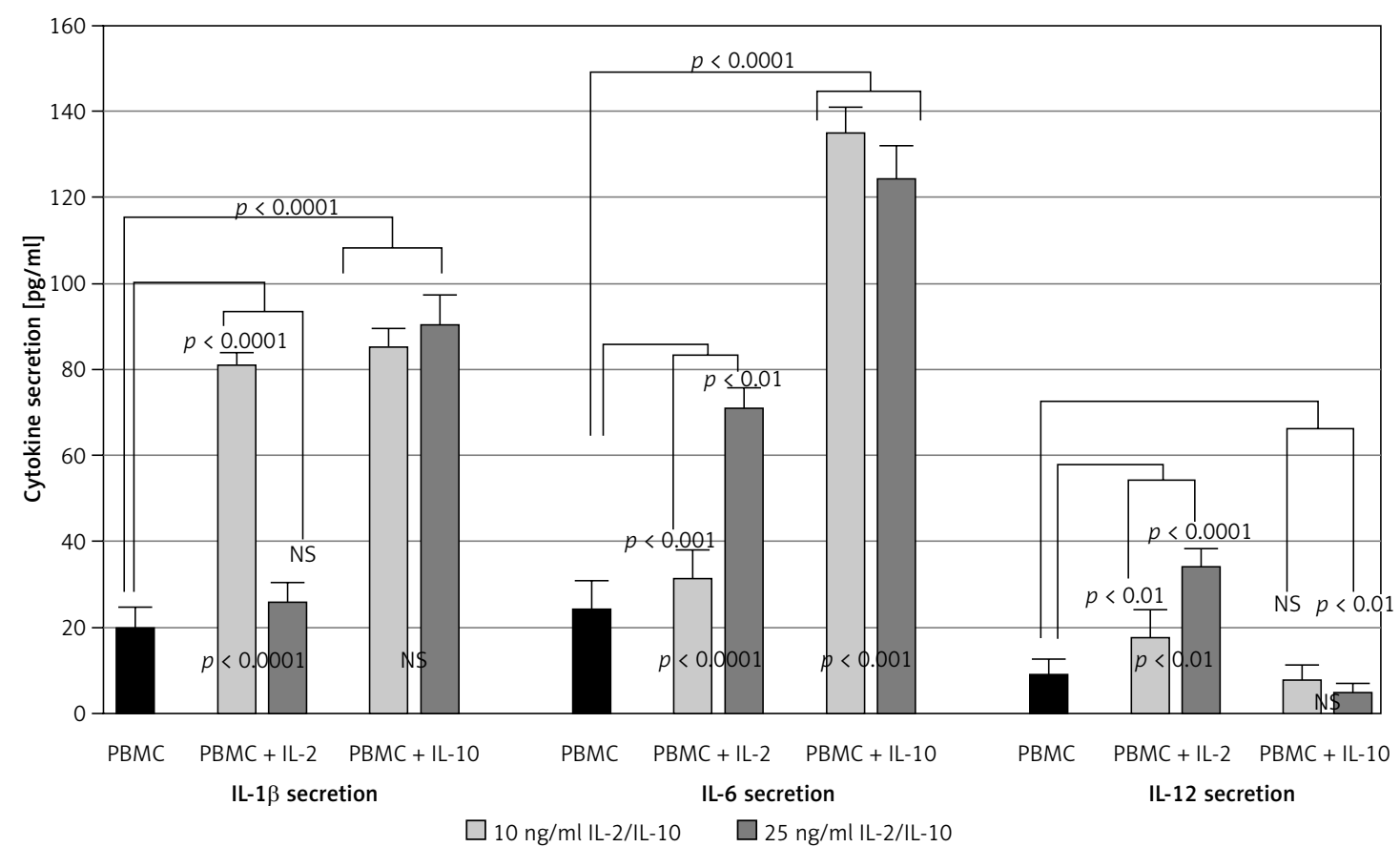

Figure 2. IL-1 $\beta$, IL-6 and IL-12 secretion by IL-2 or IL-10 activated PBMCS

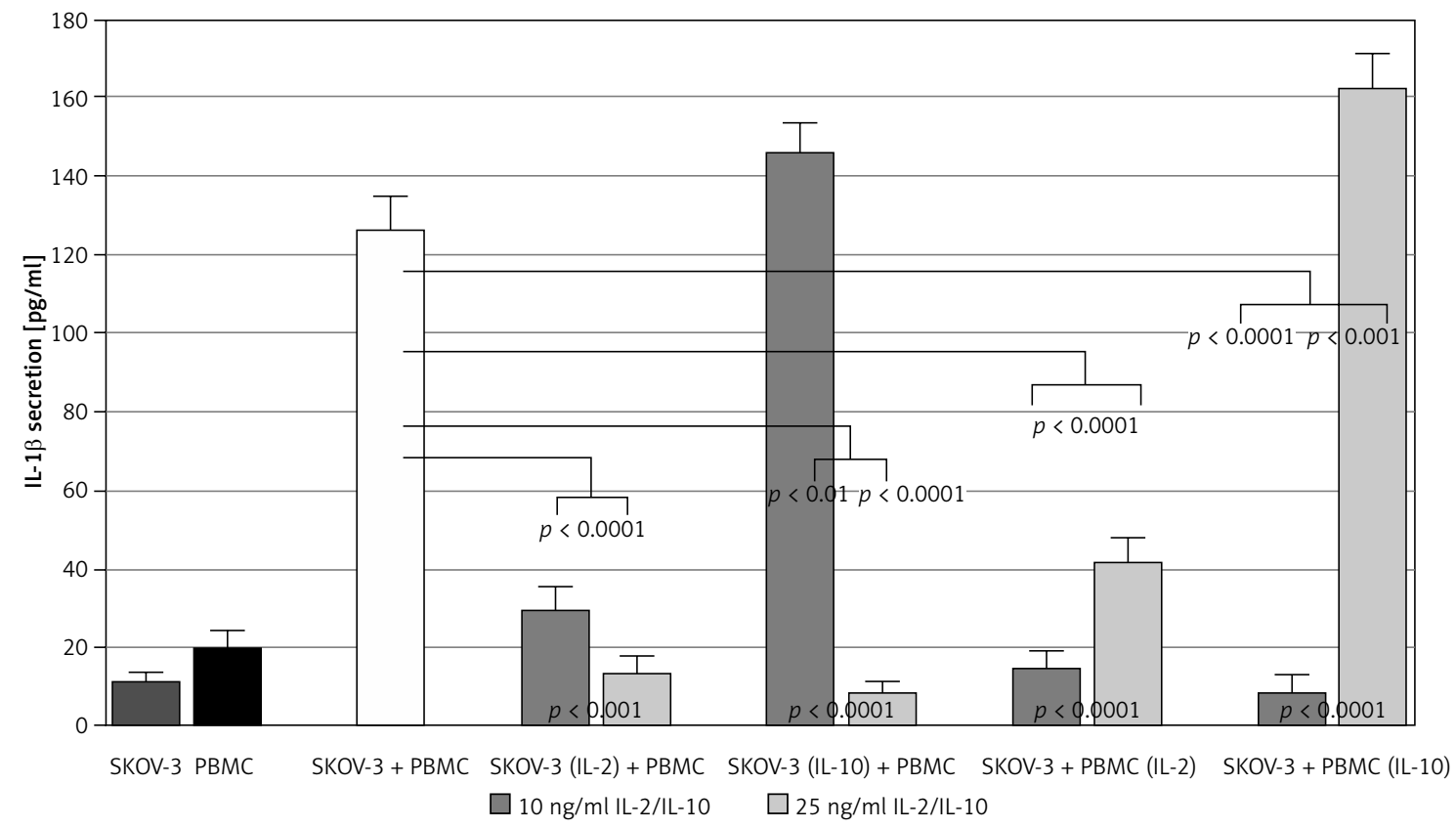

Figure 3. IL-1 $\beta$ secretion by IL-2 or IL-10 activated co-cultured SKOV-3 cells and PBMCS

\section{IL-1, IL-6 and IL-12 secretion by stimulated co-culture}

Unstimulated co-culture of SKOV-3 and PBMCs secreted more IL-1 $\beta$ than IL-6 and IL-12. In coculture when both SKOV-3 cells and PBMCs were pre-stimulated with $\mathrm{IL}-2$, production of $\mathrm{IL}-1 \beta$ $(p<0.0001)$ and IL-6 $(p<0.0001)$ was lower, but secretion of IL-12 $(p<0.001)$ was higher in comparison to the basal co-culture. A higher dose of $\mathrm{IL}-2$ resulted in the co-culture producing signifi- cantly more IL-1 $\beta(p<0.0001)$ and IL-12 $(p<0.01)$ and less IL-6 $(p<0.001)$.

Additionally, in co-culture when both SKOV-3 cells and PBMCs were pre-stimulated with $\mathrm{IL}-10$, production of $\mathrm{IL}-1 \beta(p<0.0001)$ and $\mathrm{IL}-6$ $(p<0.0001)$ was lower, but secretion of IL-12 remained at a similar level in comparison to the basal co-culture. A higher dose of IL-10 resulted in producing significantly less IL-1 $\beta$ ( $p<0.0001)$ and slightly more IL-6 $(p<0.05)$ and IL-12 $(p<0.01)$. Results for all studied cytokine secre- 


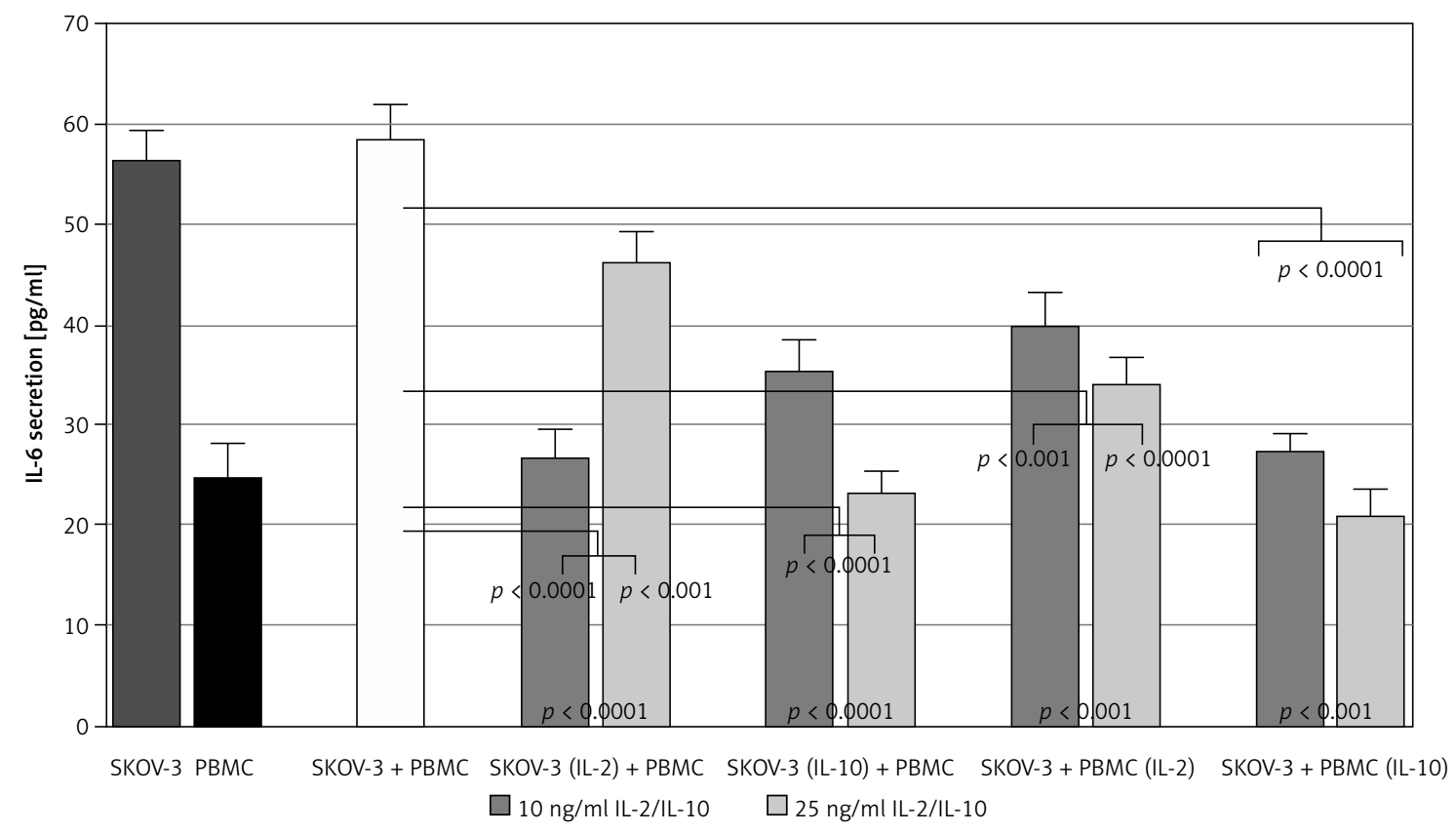

Figure 4. IL-6 secretion by IL-2 or IL-10 activated co-cultured SKOV-3 cells and PBMCS

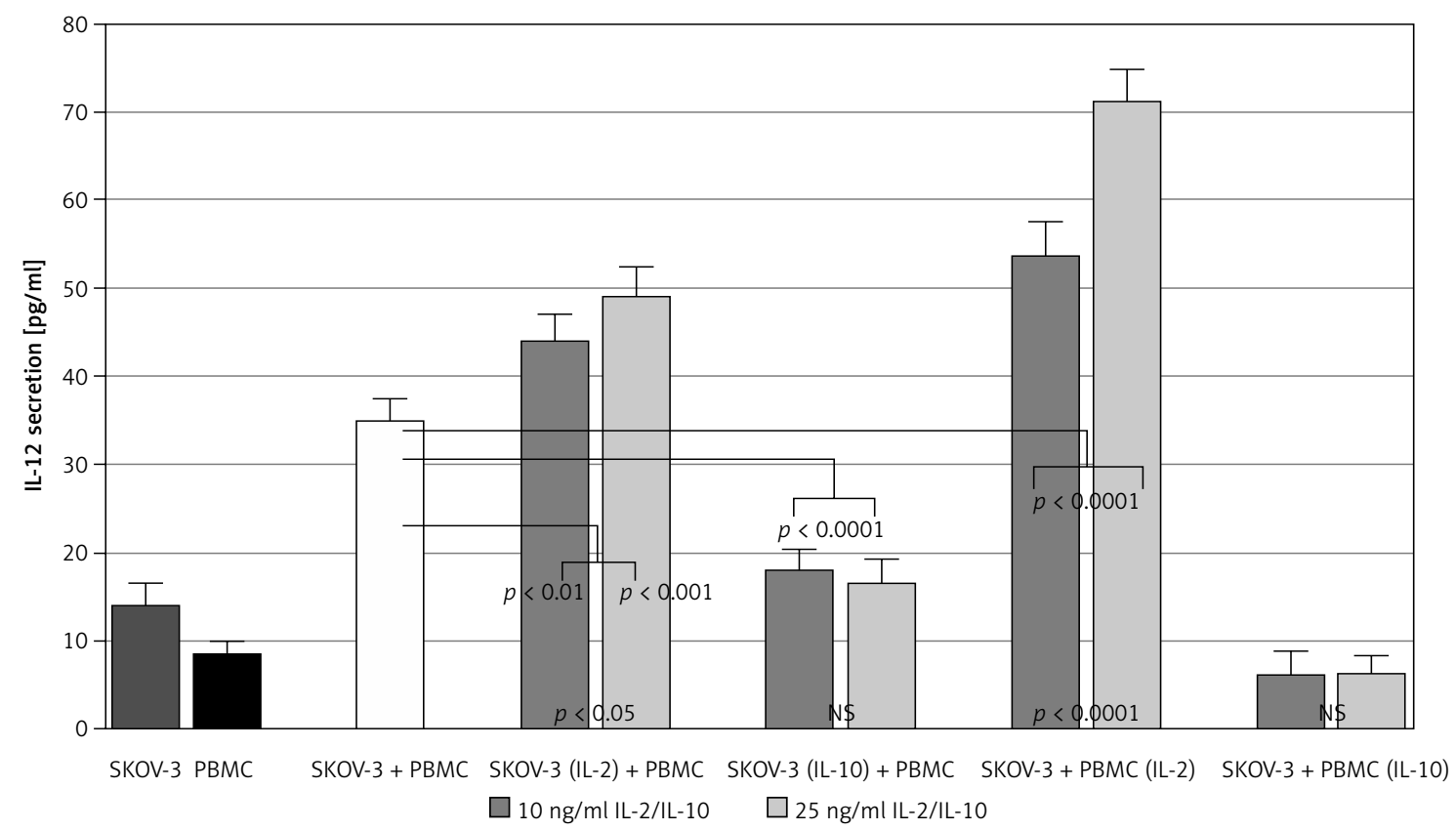

Figure 5. IL-12 secretion by IL-2 or IL-10 activated co-cultured SKOV-3 cells and PBMCS

tion by co-cultures of SKOV-3 cells and PBMCs are shown in Figures $6 \mathrm{~A}$ and $6 \mathrm{~B}$.

\section{Discussion}

Ovarian cancer is an immunogenic tumor, indicated by the presence of immune cells, especially of lymphocyte Th and Tc, dendritic cells and macrophages, which correlate positively and strongly with patient survival [14]. These cells interact directly and indirectly with cancer cells, causing a variety of biological effects, from the destruction of tumor cells through to promoting their survival. Cytokines, as a secretory product of immune cells, take part in malignant transformation, including promotion of metastasis, increasing cell adhesiveness, enhancing tumor angiogenesis and inducing the immune system to block cell-mediated mechanisms for identifying and destroying tumor cells [15]. Different cytokines, including proinflammatory factors, such as IL-1 $\beta$ and IL-6 or IL-12, were demonstrated in normal ovarian tissue and were more pronounced in tumor cells, especially in ovarian cancer cells [16]. 
A

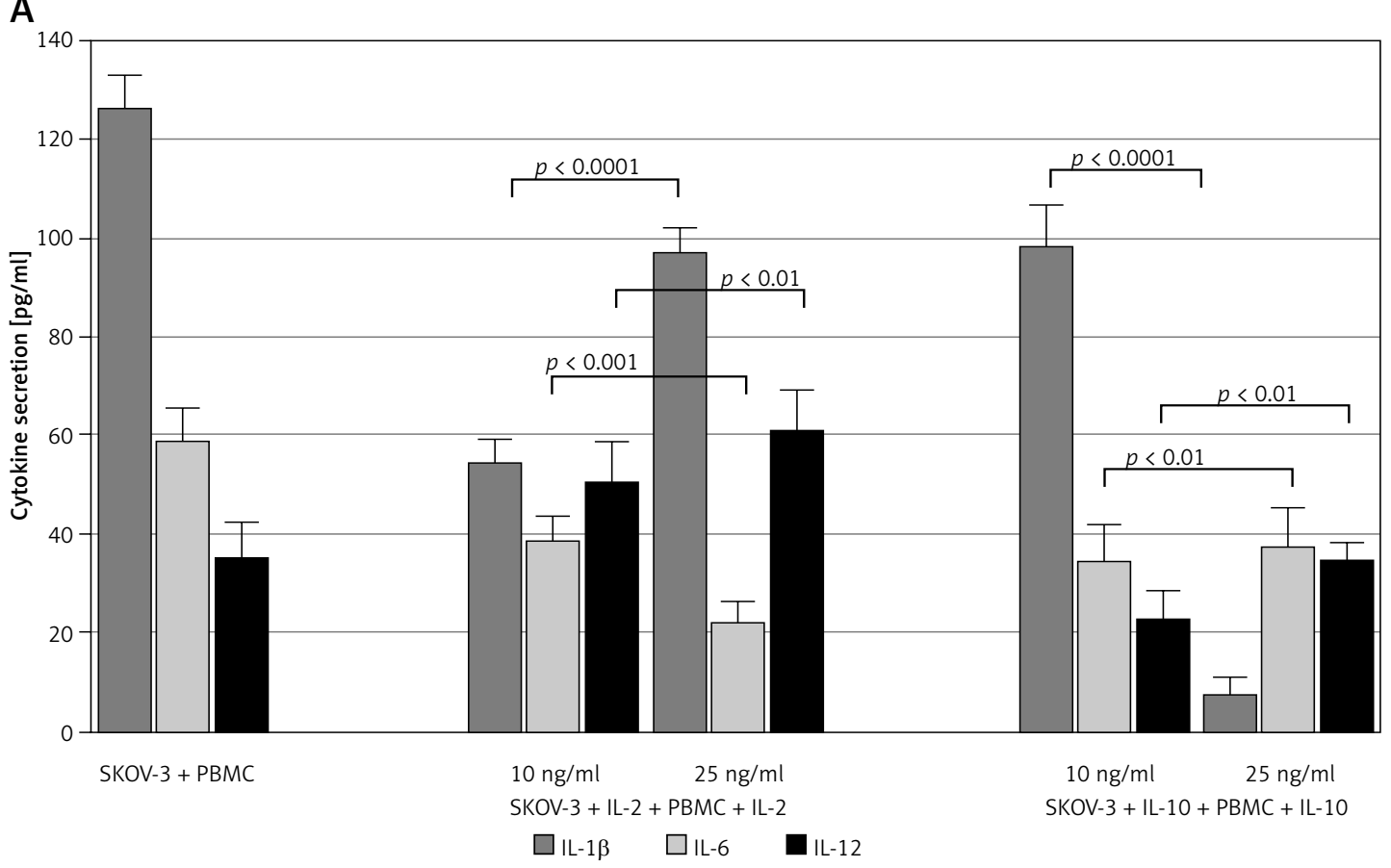

B

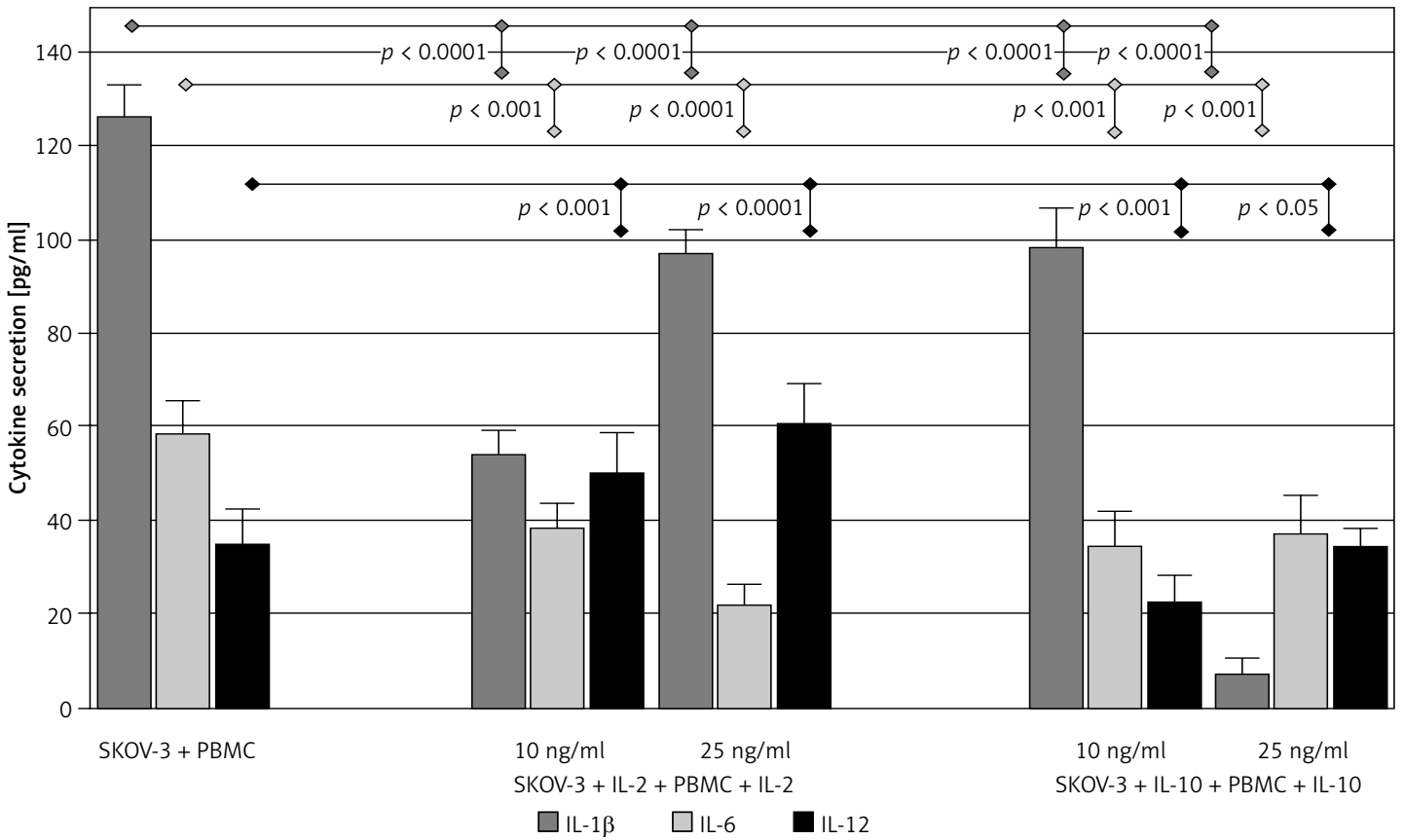

Figure 6. A - Cytokine secretion by IL-2 or IL-10 activated co-cultured SKOV-3 cells and PBMCs. B - Comparison of cytokine level after stimulation by IL-2 or IL-10 co-culture SKOV-3 cells and PBMC

The mechanism by which the immune system may prevent carcinogenesis is still poorly understood. However, well-functioning immunity counteracts cancer cell proliferation. One mechanism which is involved in tumor genesis maintenance is the proposed switch from the Th1 response profile to the Th2 profile. The Th1 response is promoted by signal transducer of Stat1 and Stat4 signaling, while a Th2 response is promoted by Stat 6 signaling. Interestingly, Th1 responses, driven by IL-2 and IL-12, are typically anticarcinogenic activity $[17,18]$. The more that the cancer cells, including SKOV-3, can secrete cytokines and soluble receptors and in this way, modulate the antitumor response [3]. Moreover, modulation of IL-1 or IL-6 expression or its related signaling pathway may be a promising strategy of treatment for drug-resistant ovarian cancer.

The most important cytokines which take part in the immune and inflammatory response are 
IL-1 $\beta$, IL-6 and IL-12. They are mostly produced by PBMCs, but recently studies strongly suggested that they are also produced by tumor cells [19]. IL-1, IL-6 and IL-12 might associate with inflammatory processes in a tumor progression and in consequence create a specific microenvironment for growth of the tumor $[20,21]$. The results of our study showed that SKOV-3 cells secreted all of the investigated cytokines. However, the secretion of IL- 6 was definitely higher than IL-1 $\beta$ and IL- 12 . After stimulation of IL-2 or IL-10, cancer cells produced more IL-1 $\beta$ and less IL- 6 , but only stimulation with IL-2 increased IL-12 production. PBMCs in basal and stimulated culture produced most of all IL-6 and least IL-12. In addition, in the cancer environment some elements of the immune system, such as PBMCs, can affect the cytokine secretion profile. Hence, our results showed that presence of PBMCs may influence especially IL-1 $\beta$ secretion. Combination of SKOV-3 and PBMC cultures in co-culture caused a significant increase of IL-1 $\beta$, especially in the basal culture of SKOV-3 cells and PBMCs. The pre-stimulation cell culture showed that IL-10 strongly influenced IL-1 $\beta$ secretion. These results suggested a significant effect of balanced Th1/Th2 on IL-1 $\beta$ secretion by SKOV-3 cells. In the case of IL-6, regardless of the model of culture, secretion of IL- 6 varied slightly or even decreased. This is a particularly surprising effect of IL-10 on IL- 6 production. This strange result showed that sometimes lower concentrations of certain cytokines can increase the secretion of various protein. However, based on our research, we are not able to state what mechanism worked here. Moreover, production of IL-12, as the most important cytotoxic cytokine, in basal and IL-2 pre-stimulated co-culture significantly increased. Our result showed that the highest IL- $1 \beta$ and IL- 6 secretion was in the basal co-culture of SKOV-3 cells and PBMCs, but adding to the culture IL-2 causes greater production of IL-12. Generally, stimulation with IL-2 assisted cytokine secretion more than IL-10.

In this investigation, we tried to explain relationships and correlations between ovarian cancer cells and elements of the immune system as well as how exogenous cytokines can effect secretion of proinflammatory cytokines by tumor cells. When choosing which dose of cytokine stimulated cell cultures, we relied on the results obtained in the work of Ingersoll et al. [3]. However, most reports referred to IL-6. Many other authors have shown that the increased secretion of IL- 6 is a characteristic of ovarian cancer cells. In vitro experiments have shown that epithelial ovarian cancer cells constitutively produce IL-6, thus modulating the host immune response to ovarian cancer [22, 23]. Moreover, increased IL-6 receptor $\alpha$ (IL-6R $\alpha$ ) expression and constitutive STAT3 activation also have been found to associate with ovarian cancer proliferation [24]. IL-6, as an important proinflammatory cytokine, affects many mechanisms in the tumor microenvironment, including inflammation and recruitment of neutrophils, and promotes the migration and proliferation of $T$ lymphocytes and changing the Th1/Th2 balance. Findings also show that IL-6 provides an important signal in the development of Th17 cells while blocking the differentiation of T CD4+ cells into T regulatory (Treg) cells [25]. The results of Takaishi et al. [23] indicate that Stat3 activation by IL-6 plays an important role in cellto-cell interaction between tumor cells and $M 2$ macrophages in the ascites of advanced epithelial ovarian cancer. Moreover, higher IL-6 serum concentration is associated with negative prognoses in independent cancer types, and it seems to be associated with tumor size and stage or disease progression [25, 26]. Findings of Wang et al. [27] suggest that the production of IL- 6 protects the cells from cytotoxic agents through down-regulation of proteolytic activation of caspase-3, and the expression level of IL- 6 is positively associated with their degree of chemoresistance in ovarian cancer cells. The autocrine production level of IL-6 by the SKOV-3 cell line is inversely associated with their response to cisplatin and paclitaxel [27]. The ovarian cancer cell line SKOV-3 cultured with IL-6 has increased chemotactic and/or chemokinetic activity and increased overall invasiveness. Moreover, IL- 6 has been reported to be a potent proangiogenic cytokine in ovarian cancer cells [28]. Studies demonstrated that IL-6 may contribute to ovarian cancer cell growth partly through the activation of androgen receptor (AR) and estrogen receptor (ER) pathways $[29,30]$. However, not all results confirm that the SKOV-3 cells are able to produce IL-6. Kulbe et al. [31] did not find secretion of IL- 6 by SKOV-3 cells, although the experimental design was very similar to that used by other authors. These differences may be due to the presence of complex forms of IL- 6 in the supernatants of cell cultures, which cannot be detected by ELISA.

Carcinogenesis is associated with inflammation, and IL-1 $\beta$ is the most important cytokine involved in this process. IL-1 $\beta$ is secreted mainly by monocytes and macrophages, but studies indicated that it is also secreted by malignant cells, including ovarian cancer cells, which was also confirmed by our research. Investigations of other authors suggest that not all cell lines of ovarian cancer are able to secrete IL-1 $\beta$. Li et al. [32] evaluated the ability of 5 ovarian cancer cell lines to produce IL-1 $\beta$ : OVCAR-3, OVCA 420, OVCA 429, OVCA 433 and DOV 13. The results showed that 
4 cell lines expressed mRNA for IL-1 $\beta$, but only 3 of them secreted $\mathrm{IL}-1 \beta$ protein to the culture medium. These results showed that mRNA expression is not always related to protein production.

In our study, we also showed that an imbalance in Th1/Th2 response can affect the secretory ability of SKOV-3 cells to produce proinflammatory cytokine. Moreover, cytokine-derived Th cells can also influence proliferation of malignant cells. Despite the fact that these two cytokines are not the main representatives of groups Th1 and Th2, we deliberately used them in the experiment. The main reason was that both IL-2 and IL-10 are taken into consideration in the treatment of neoplastic diseases in the treatment of ovarian cancer. Anti-cancer properties are assigned to IL-2, and it has begun to be used in treatment of metastatic renal cancer. Its application in other tumors, including ovarian cancer, is a major concern. Clinical studies have shown that IL-2 could increase the remission period in ovarian cancer. The major problem is adjustment of the appropriate dose of this cytokine to limit its toxic effect in patients [33]. Studies conducted on mice, which had implanted ovarian neoplasms, showed that IL-2 administration directly into the tumor increased animals' survival and delayed tumor development [34]. Moreover, the cytotoxicity of IL-2 in combination with PBMCs against ovarian cancer was evaluated. Two ovarian cancer cell-lines (SKOV-3 and CAR3) were transfected with a plasmid encoding red fluorescent protein (RFP). Cytotoxicity of SKOV-3-RFP cells mediated by PBMCs and cytokines was determined by lactate dehydrogenase release. PBMCs in combination with IL-2 showed clear dose-dependent cytotoxicity against SKOV-3-RFP [3, 35]. Use of IL-2 in our study showed decreased levels of both investigated cytokines in supernatants from co-cultures. It is not surprising, because IL-2 is known to be an immunomodulating cytokine and it can inhibit the proinflammatory reaction. In the case of IL-10, the situation is much more complicated. The influence of IL-10 on the immune system is an object of interest of many investigators. The secretion of IL-10 by normal and cancerous ovarian cells was proved in experiment with ovarian tissue homogenates. ELISA examination revealed that IL-10 protein was expressed in all 31 normal and 25 cancerous ovarian tissue homogenates. The results show that both normal and cancerous ovarian tissues produce and secrete IL-10. IL-10 protein levels were significantly higher in normal ovarian tissues compared to cancerous tissues [36]. Over the years it was thought that IL-10 is an immunosuppressive cytokine, but studies have shown that under certain conditions it may also function as an immunostimulatory agent. More than a decade after its original description, the source of IL-10 in ovarian carcinoma, and its role in the cell and tumor micro-environment, remain unresolved. Studies conducted by Groux et al. [37] and Santin et al. [38] have shown that IL-10 can act as a stimulator of cytotoxic activity of $\mathrm{T}$ lymphocytes and increase secretion of Th1 profile cytokines such as IL-2 and IFN- $\gamma$ in CD8+ lymphocytes. In this investigation, we observed an ambiguous influence of IL-10 on proinflammatory cytokine secretion. IL-10 was used for stimulation of ovarian cancer cell lines, and the results showed a very interesting effect in one type of co-culture. In co-cultures in which only PBMCs were stimulated with IL-10, a lower concentration of IL-10 caused a statistically significant decrease of IL-1 $\beta$ secretion, but a higher concentration of IL-10 used for stimulation caused a large increase of IL-1 $\beta$ secretion. Many types of neoplastic cells are able to secrete cytokines, which facilitate their growth or metastasis or promote tumor cell survival under unfavorable conditions. Cytokines secreted by tumor cells affect immune system decreasing its ability to eliminate neoplastic cells. But not only tumor cells are responsible for secretion of cytokines, which favors tumor survival. There are Treg lymphocytes, which were shown to play an important role in cross-talk between the tumor and the immune system. The T cell/Treg cell ratio is lower in ascites from patients with an advanced stage of ovarian carcinoma. The same team reported that Treg depletion reduced tumor weight in an animal model of ovarian carcinoma. In addition, the cytokine profile in animals changed to one more supporting of anti-cancer immunity. These findings showed that not only proteins such as cytokines or tumor cells can be the target for new therapeutic strategies of ovarian cancer [39]. Our experiment also showed that unstimulated PBMCs have the ability to produce investigated cytokines, and after stimulation, the level of cytokines is changed. Recently it was shown that not only cells or cytokines affect the anti-cancer immune response [39, 40]. Also immune cells and mesenchymal cells can interact with malignant cells. Wang et al. [41] showed that the cross talk between ovarian cancer cells and tumor-associated macrophages enhances the ability of endothelial cells to promote the progression of ovarian cancer. Moreover, Lis et al. [42] demonstrated that co-culture of ovarian cancer cells in direct cellular contact with mesenchymal stem cells induces broad transcriptomic changes related to cellular adhesion, invasion, migration, proliferation and chemoresistance.

In conclusion, all these data indicate that the interactions between the immune system and tumor cells are complicated and mediated via cells, cytokines and other components of the microenvi- 
ronment. Our results suggested that Th1/Th2 type of cytokines might influence SKOV-3 ovarian cells' pro-inflammatory activation. Co-cultures ofSKOV-3 cells and PBMCs showed significant changes in cross-talk between cancer and immune cells. That observation can be useful in understanding the pathogenesis of ovarian cancer. This identifies the need to create composite therapies targeting many different mechanisms. Moreover, the imbalance in Th1/Th2 response could be a target in biological treatment for modulation of inflammation during ovarian cancer.

\section{Conflict of interest}

Patrycja Mickiewicz is supported by the Forszt project co-financed by the EU from the European Social Fund.

None of the authors has any proprietary, financial, professional or other personal interest of any nature or kind in any product, service and/or company.

\section{References}

1. Jemal A, Tiwari RC, Murray T. Cancer statistics. CA Cancer J Clin 2004; 54: 8-29.

2. Gottwald L, Danilewicz M, Fendler W, et al. The AgNORs count in predicting long-term survival in serous ovarian cancer. Arch Med Sci 2014; 10: 84-90.

3. Ingersoll SB, Patel S, Caballero L, et al. Synergistic cytotoxicity of interferon alpha-2b and interleukin-2 in combination with PBMC against ovarian cancer: development of an experimental model for cellular therapy. Gynecol Oncol 2009; 112: 192-8.

4. Li J, Fadare O, Xiang L, Kong B, Zheng W. Ovarian serous carcinoma: recent concepts on its origin and carcinogenesis. J Hematol Oncol 2012; 5: 8.

5. Kandalaft LE, Motz GT, Duraiswamy J, Coukos G. Tumor immune surveillance and ovarian cancer: lessons on immune mediated tumor rejection or tolerance. Cancer Metastasis Rev 2011; 30: 141-51.

6. Gómez-Raposo C, Mendiola M, Barriuso J, Casado E, Hardisson D, Redondo A. Angiogenesis and ovarian cancer. Clin Transl Oncol 2009; 11: 564-71.

7. Gavalas NG, Karadimou A, Dimopoulos MA, Bamias A. Immune response in ovarian cancer: how is the immune system involved in prognosis and therapy: potential for treatment utilization. Clin Dev Immunol 2010; 2010: 791603.

8. Bellati F, Visconti V, Napoletano C, et al. Immunology of gynecologic neoplasms: analysis of the prognostic significance of the immune status. Curr Cancer Drug Targets 2009; 9: 541-65.

9. Kusuda T, Shigemasa K, Arihiro K, Fujii T, Nagai N, Ohama K. Relative expression levels of Th1 and Th2 cytokine mRNA are independent prognostic factors in patients with ovarian cancer. Oncol Rep 2005; 13: 1153-8.

10. Sykes L, MacIntyre DA, Yap XJ, Teoh TG, Bennett PR. The Th1:th2 dichotomy of pregnancy and preterm labour. Mediators Inflamm 2012; 2012: 967629.

11. Lowe DB, Storkus WJ. Chronic inflammation and immunologic-based constraints in malignant disease. Immunotherapy $2011 ; 3: 1265-74$.
12. Fridman WH, Galon J, Dieu-Nosjean MC, et al. Immune infiltration in human cancer: prognostic significance and disease control. Curr Top Microbiol Immunol 2011; 344: 1-24.

13. Ellyard JI, Simson L, Parish CR. Th2-mediated anti-tumour immunity: friend or foe? Tissue Antigens 2007; 70: 1-11.

14. Lindau D, Gielen P, Kroesen M, Wesseling P, Adema GJ. The immunosuppressive tumour network: myeloid-derived suppressor cells, regulatory T cells and natural killer T cells. Immunology 2013; 138: 105-15.

15. Finn OJ. Immunooncology: understanding the function and dysfunction of the immune system in cancer. Ann Oncol 2012; 23 Suppl. 8: viii6-9.

16. Hao NB, Lü MH, Fan YH, Cao YL, Zhang ZR, Yang SM. Macrophages in tumor microenvironments and the progression of tumors. Clin Dev Immunol 2012; 2012: 948098.

17. Liao W, Lin JX, Leonard WJ. Interleukin-2 at the crossroads of effector responses, tolerance, and immunotherapy. Immunity 2013; 38: 13-25.

18. Chapoval S, Dasgupta P, Dorsey NJ, Keegan AD. Regulation of the $T$ helper cell type 2 (Th2)/T regulatory cell (Treg) balance by IL-4 and STAT6. J Leukoc Biol 2010; 87: 1011-8.

19. Apte RN, Voronov E. Is interleukin-1 a good or bad 'guy' in tumor immunobiology and immunotherapy? Immunol Rev 2008; 222: 222-41.

20. Dinarello CA. Why not treat human cancer with interleukin-1 blockade? Cancer Metastasis Rev 2010; 29: 317-29.

21. Sansone P, Bromberg J. Targeting the interleukin-6/Jak/ stat pathway in human malignancies. J Clin Oncol 2012; 30: 1005-14.

22. Chou $\mathrm{CH}$, Wei LH, Kuo ML, et al. Up-regulation of interleukin-6 in human ovarian cancer cell via a Gi/PI3K-Akt/ NF-kappaB pathway by lysophosphatidic acid, an ovarian cancer-activating factor. Carcinogenesis 2005; 26: 45-52.

23. Takaishi K, Komohara Y, Tashiro $\mathrm{H}$, et al. Involvement of M2-polarized macrophages in the ascites from advanced epithelial ovarian carcinoma in tumor progression via Stat3 activation. Cancer Sci 2010; 101: 2128-36.

24. Syed V, Ulinski G, Mok SC, Ho SM. Reproductive hormone-induced, STAT3-mediated interleukin 6 action in normal and malignant human ovarian surface epithelial cells. J Natl Cancer Inst 2002; 94: 617-29.

25. Lippitz BE. Cytokine patterns in patients with cancer: a systematic review. Lancet Oncol 2013; 14: e218-28.

26. Kovacs E. Interleukin-6 leads to interleukin-10 production in several human multiple myeloma cell lines. Does interleukin-10 enhance the proliferation of these cells? Leuk Res 2010; 34: 912-6.

27. Wang Y, Niu XL, Qu Y, et al. Autocrine production of interleukin- 6 confers cisplatin and paclitaxel resistance in ovarian cancer cells. Cancer Lett 2010; 295: 110-23.

28. Nilsson MB, Langley RR, Fidler IJ. Interleukin-6, secreted by human ovarian carcinoma cells, is a potent proangiogenic cytokine. Cancer Res 2005; 65: 10794-800.

29. Wang Y, Yang J, Gao Y, Dong LJ, Liu S, Yao Z. Reciprocal regulation of 5 alpha-dihydrotestosterone, interleukin- 6 and interleukin-8 during proliferation of epithelial ovarian carcinoma. Cancer Biol Ther 2007; 6: 864-71.

30. Yang J, Wang Y, Gao Y, Shao J, Zhang XJ, Yao Z. Reciprocal regulation of 17 beta-estradiol, interleukin- 6 and interleukin-8 during growth and progression of epithelial ovarian cancer. Cytokine 2009; 46: 382-91. 
31. Kulbe, H, Thompson R, Wilson JL, et al. The inflammatory cytokine tumor necrosis factor-alpha generates an autocrine tumor-promoting network in epithelial ovarian cancer cells. Cancer Res 2007; 67: 585-92.

32. Li BY, Mohanraj D, Olson MC, et al. Human ovarian epithelial cancer cells cultured in vitro express both interleukin 1alpha and beta genes. Cancer Res 1992; 52: 2248-52.

33. Wei S, Kryczek I, Edwards RP, et al. Interleukin-2 administration alters the CD4+FOXP3+ T-cell pool and tumor trafficking in patients with ovarian carcinoma. Cancer Res 2007; 67: 7487-94.

34. Jackaman C, Bundell CS, Kinnear BF, et al. IL-2 intratumoral immunotherapy enhances CD8 T cells that mediate destruction of tumor cells and tumor-associated vasculature: a novel mechanism for IL-2. J Immunol 2003; 171: 5051-63.

35. Ingersoll SB, Ahmad S, Stoltzfus GP, et al. Functional characterization of a fluorescent highly tumorigenic ovarian cancer line to test cellular therapy in experimental models. Int J Gynecol Cancer 2011; 21: 457-65.

36. Rabinovich A, Medina L, Piura B, Segal S, Huleihel M. Regulation of ovarian carcinoma SKOV-3 cell proliferation and secretion of MMPs by autocrine IL-6. Anticancer Res 2007; 27 (1A): 267-72.

37. Groux H, Bigler M, de Vries JE, Roncarolo MG. Inhibitory and stimulatory effects of IL-10 on human CD8 + T cells. J Immunol 1998; 160: 3188-93.

38. Santin AD, Hermonat PL, Ravaggi A, et al. Interleukin-10 increases Th1 cytokine production and cytotoxic potential in human papillomavirus-specific CD8(+) cytotoxic T lymphocytes. J Virol 2000; 74: 4729-37.

39. Chen F, Hou M, Ye F, Lv W, Xie X. Ovarian cancer cells induce peripheral mature dendritic cells to differentiate into macrophage like cells in vitro. Int J Gynecol Cancer 2009; 19: 1487-93.

40. Chen LL, Ye F, Lü WG, Yu Y, Chen HZ, Xie X. Evaluation of immune inhibitory cytokine profiles in epithelial ovarian carcinoma. J Obstet Gynaecol Res 2009; 35: 212-8.

41. Wang X, Zhao X, Wang K, Wu L, Duan T. Interaction of monocytes/macrophages with ovarian cancer cells promotes angiogenesis in vitro. Cancer Sci 2013; 104: 516-23.

42. Lis R, Touboul C, Halabi NM, et al. Mesenchymal cell interaction with ovarian cancer cells induces a back ground dependent pro-metastatic transcriptomic profile. J Transl Med 2014; 12: 59. 